\title{
COVID-19 in IgA Vasculitis
}

\author{
Mahdieh Sadat Mousavi (ib) ${ }^{1, *}$ and Mohsen Jafari ${ }^{2}$ \\ ${ }^{1}$ Department of Pediatric Rheumatology, Bahrami Children's Hospital, Tehran University of Medical Sciences, Tehran, Iran \\ ${ }^{2}$ Department of Pediatric Infectious Diseases, Bahrami Children's Hospital, Tehran University of Medical Sciences, Tehran, Iran \\ Corresponding author: Bahrami Children’s Hospital, Shahid Kiai St, Damavand Ave, Tehran, Iran. Tel: +98-2177568809, Fax: +98-21775688, Email: mousavi1387@yahoo.com
}

Received 2020 May 04; Revised 2020 June 25; Accepted 2020 June 28.

Keywords: Vasculitis, IgA, COVID-19

\section{Dear Editor,}

The coronavirus disease 2019 (COVID-19), classified as a pandemic by the World Health Organization (WHO), has affected nearly all countries and regions around the world. Evidence to date suggests that patients with concomitant medical conditions are at higher risk for severe COVID19 (1). However, data regarding the comorbidities in children with COVID-19 is relatively rare (2). We report a case of suspected COVID-19 infection in a 6-year-old boy with Henoch-Schonlein purpura (HSP) complicated by diffuse alveolar hemorrhage.

On April 1, 2020, the patient was brought to the emergency department of Bahrami Children's Hospital, Tehran, Iran, because of a palpable purpuric maculopapular rash involving his left ankle and foot and signs of arthritis in the left ankle and knee. The rest of the physical examination was unremarkable. The child was admitted for further evaluation. Laboratory tests showed leukocytosis, increased ESR and CRP and mild hematuria and proteinuria. The patient was clinically diagnosed with HSP, was given supportive treatment and ibuprofen. Arthralgia and edema gradually subsided and the patient was discharged on day 3 with a prescription of ibuprofen.

Two days later, the patient was readmitted with fever, tachypnea, dyspnea, $\mathrm{O} 2$ saturation of $94 \%$ on room air, bilateral fine rales on lung auscultation and abdominal pain associated with melena. Patient's hemoglobin was decreased and urine analysis showed hematuria and proteinuria. Abdominal ultrasound demonstrated mural thickening of distal ileum and decreased peristalsis. Chest X-ray (CXR) showed bilateral, peripheral ground glass opacities of the lungs. COVID-19 rapid test was negative; however, a clinical diagnosis of COVID-19 was made and after PICU admission treatment started with methyl prednisolone, cloxacillin, azithromycin and hydroxychloroquine. As patient's condition worsened and his hemoglobin level dropped to $6.1 \mathrm{gr} / \mathrm{dL}$, packed red cells were given. COVID-19 rapid test repeat revealed negative results. The patient was suspected to have developed pulmonary hemorrhage associated with HSP. Bronchoscopy and bronchoalveolar lavage (BAL) showed diffuse alveolar hemorrhage. As BAL specimen tested negative for SARS-CoV-2, the patient received intravenous cyclophosphamide. However, treatment was not successful and the patient died of respiratory failure.

COVID-19 is a major concern to rheumatologists as rheumatologic patients may potentially be at an increased risk of infection and death due to the underlying immune dysfunction and treatment with immunosuppressive agents (3). However, data regarding rheumatologic diseases as a risk factor for increased incidence or severity of COVID-19 in children is very scarce (4).

Few reports have described adults and children presenting with maculopapular, purpuric and acro-ischemic skin lesions who were subsequently diagnosed with COVID-19 (5, 6). Moreover, vasculitis-like lesions due to endothelial damage are reported in severe forms of COVID-19 (7). At first presentation, our patient had the typical palpable purpuric skin lesions of HSP and his symptoms were successfully managed with ibuprofen. However, he was readmitted with pulmonary manifestations, which led to a clinical diagnosis of COVID-19, and exacerbation of HSP-related symptoms.

Despite negative COVID-19 rapid test, our primary treatment strategy was to manage both HSP and COVID-19 manifestations, i.e. methyl prednisolone, antibiotics and hydroxychloroquine. With worsening of respiratory manifestations, unexplained decline of hemoglobin level led us to perform bronchoscopy and BAL, which confirmed diffuse alveolar hemorrhage, a rare complication of HSP which manifests with tachypnea, dyspnea, sudden drop of hemoglobin and diffuse pulmonary infiltrates and is associated with poor prognosis (8). 
During hospitalization, our patient was tested three times for COVID-19, one taken from the BAL specimen. It is believed that severe respiratory distress met in critically-ill COVID-19 patients results from a cytokine storm syndrome, a condition which may also complicate rheumatologic diseases, including vasculitis. At the time of the epidemic, patients presenting with severe respiratory manifestations and underlying vasculitis should be suspected of COVID-19 despite a primary negative COVID-19 rapid test.

This report doesn't draw any conclusions on the possible association between pediatric rheumatologic conditions and COVID-19. Obviously, all children suspected with COVID-19 should be managed according to the standard protocols but it is mandatory to investigate other differential diagnoses, particularly in patients with underlying conditions. In case of concurrent COVID-19 and a rheumatologic condition, it seems reasonable to choose a treatment plan to manage both conditions.

Clinical judgment and decision making about vasculitis is challenging in COVID-19 pandemic due to:

1) COVID-19 can mimic cutaneous, pulmonary and renal manifestations of vasculitis.

2)COVID-19 can trigger some vasculitic signs and symptoms.

3) Vasculitis treatment influenced by COVID-19 pandemic while sensitivity and specificity of RT-PCR and COVID-19 serology are unclear.

\section{Footnotes}

Authors' Contribution: Mahdieh Sadat Mousavi did drafting of the manuscript and managing the patient. Mohsen Jafari did managing the patient.
Conflict of Interests: No conflict of Interest. Funding/Support: Not declared by author.

\section{References}

1. Yang J, Zheng Y, Gou X, Pu K, Chen Z, Guo Q, et al. Prevalence of comorbidities and its effects in patients infected with SARS-CoV-2: A systematic review and meta-analysis. Int J Infect Dis. 2020;94:91-5. doi: 10.1016/j.ijid.2020.03.017. [PubMed: 32173574]. [PubMed Central: PMC7194638].

2. Ludvigsson JF. Systematic review of COVID-19 in children shows milder cases and a better prognosis than adults. Acta Paediatr 2020;109(6):1088-95. doi: 10.1111/apa.15270. [PubMed: 32202343] [PubMed Central: PMC7228328].

3. Robinson PC, Yazdany J. The COVID-19 global rheumatology alliance: Collecting data in a pandemic. Nat Rev Rheumatol. 2020;16(6):293-4 doi: 10.1038/s41584-020-0418-0. [PubMed: 32242121]. [PubMed Central: PMC7117553].

4. Haslak F, Yildiz M, Adrovic A, Barut K, Kasapcopur O. Childhood rheumatic diseases and COVID-19 pandemic: An intriguing linkage and a new horizon. Balkan Med J. 2020;37(4):184-8. doi:10.4274/balkanmedj.galenos.2020.2020.4.43. [PubMed: 32264666]. [PubMed Central: PMC7285673].

5. Landa N, Mendieta-Eckert M, Fonda-Pascual P, Aguirre T. Chilblain-like lesions on feet and hands during the COVID-19 Pandemic. Int J Dermatol. 2020;59(6):739-43. doi: 10.1111/ijd.14937. [PubMed: 32329897]. [PubMed Central: PMC7264591].

6. Mazzotta F, Troccoli T. Monday's case. Acute acro-ischemia in the child at the time of COVID-19. Dermatologia Pediatrica Bari; 2020. Available from: https://drive.google.com/file/d/ 1ehu5OlThQLwntuDiKpfKUwitxcQF-Dh6/view.

7. Zhang W, Zhao Y, Zhang F, Wang Q, Li T, Liu Z, et al. The use of anti-inflammatory drugs in the treatment of people with severe coronavirus disease 2019 (COVID-19): The perspectives of clinical immunologists from China. Clin Immunol. 2020;214:108393. doi: 10.1016/j.clim.2020.108393. [PubMed: 32222466]. [PubMed Central: PMC7102614]

8. Kamath N, Rao S. Henoch-Schonlein purpura: An update. Indian J Rheumatol. 2012;7(1):92-8. doi:10.1016/s0973-3698(12)60034-x. 